\title{
Parallel Computations of Natural Convection Flow in a Tall Cavity Using an Explicit Finite Element Method
}

\author{
T.A. Dunn, R.C. McCallen
}

This article was submitted to

First MIT Conference on Computational Fluid and Solid Mechanics, Cambridge, MA, June 12-14, 2001

\section{October 17, 2000}

U.S. Department of Energy

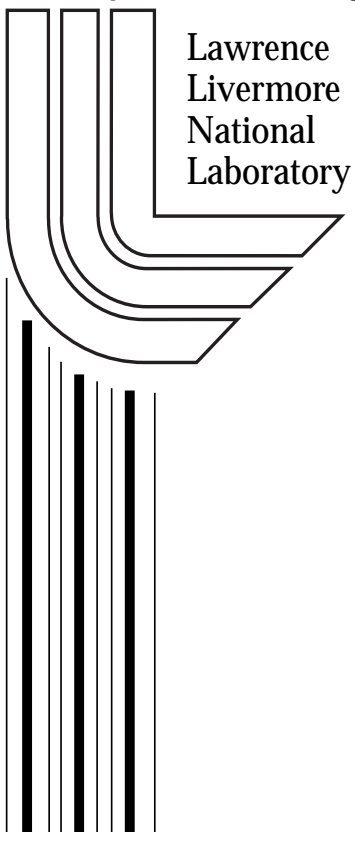




\section{DISCLAIMER}

This document was prepared as an account of work sponsored by an agency of the United States Government. Neither the United States Government nor the University of California nor any of their employees, makes any warranty, express or implied, or assumes any legal liability or responsibility for the accuracy, completeness, or usefulness of any information, apparatus, product, or process disclosed, or represents that its use would not infringe privately owned rights. Reference herein to any specific commercial product, process, or service by trade name, trademark, manufacturer, or otherwise, does not necessarily constitute or imply its endorsement, recommendation, or favoring by the United States Government or the University of California. The views and opinions of authors expressed herein do not necessarily state or reflect those of the United States Government or the University of California, and shall not be used for advertising or product endorsement purposes.

This is a preprint of a paper intended for publication in a journal or proceedings. Since changes may be made before publication, this preprint is made available with the understanding that it will not be cited or reproduced without the permission of the author.

This report has been reproduced directly from the best available copy.

Available to DOE and DOE contractors from the

Office of Scientific and Technical Information

P.O. Box 62, Oak Ridge, TN 37831

Prices available from (423) 576-8401

http:/ / apollo.osti.gov/bridge/

Available to the public from the National Technical Information Service

U.S. Department of Commerce 5285 Port Royal Rd., Springfield, VA 22161 http://www.ntis.gov/

OR

Lawrence Livermore National Laboratory Technical Information Department's Digital Library http://www.llnl.gov/tid/Library.html 


\title{
Parallel Computations of Natural Convection Flow in a Tall Cavity Using an Explicit Finite Element Method
}

\author{
Timothy A. Dunn and Rose C. McCallen \\ University of California, Lawrence Livermore National Laboratory, New Technologies \\ Engineering Division, Livermore, CA 94551, USA
}

\begin{abstract}
The Galerkin Finite Element Method was used to predict a natural convection flow in an enclosed cavity. The problem considered was a differentially heated, tall (8:1), rectangular cavity with a Rayleigh number of $3.4 \times 10^{5}$ and Prandtl number of 0.71 . The incompressible Navier-Stokes equations were solved using a Boussinesq approximation for the buoyancy force. The algorithm was developed for efficient use on massively parallel computer systems. Emphasis was on time-accurate simulations. It was found that the average temperature and velocity values can be captured with a relatively coarse grid, while the oscillation amplitude and period appear to be grid sensitive and require a refined computation.
\end{abstract}

Keywords: Incompressible Flow; Natural Convection; Boussinesq; Navier-Stokes; Finite Element Method; Cavity. 


\section{Introduction}

For introductory material and problem definitions, refer to the introduction to the First M.I.T. Conference on Computational Fluid and Solid Mechanics special session, “Computational Predictability of Natural Convection Flows in Enclosures" [1].

\section{Methodology}

An Eulerian formulation of the time-dependent three-dimensional incompressible NavierStokes equations was solved using the Galerkin finite-element method (FEM). The discretized continuity and momentum equations can be written in matrix form as $C^{T} u=0$

$M \dot{u}+(K+N(u)) u+C P=f$

where $u$ is the nodal velocity vector, $P=p / \rho, p$ is the pressure vector, $\rho$ is the fluid density, $M$ is the mass matrix, $K$ is the diffusivity, $N(u)$ is the advection operator, $C$ is the gradient operator, and $f$ is the external body force and user-supplied natural boundary condition. For more details see Gresho et al. [2].

In the current implementation, the Q1Q0 element formulation was used for 8node hexahedral brick elements. This provides tri-linear velocity interpolation in three dimensions and piecewise constant pressure. A lumped mass matrix was employed and the coefficient matrices were generated using one-point Gaussian quadrature. The continuity and momentum equations were solved simultaneously for the velocity difference and pressure with an explicit forward Euler time integration

$$
\begin{array}{lll}
M & C & v \\
C^{T} & 0 & P
\end{array}=\begin{aligned}
& F \\
& 0
\end{aligned}
$$


where $F=f-(K+N(u)) u, v=\frac{u^{n+1}+u^{n}}{\Delta t}, \Delta t$ is the time step, and $n$ is the current timestep level. The Schur compliment of Eq (3) is taken to form the equivalent pressure Poisson equation, $\left(C^{T} M^{-1} C\right) P=C^{T} M^{-1} F$.

This equation is solved for pressure, which is then back-substituted into the full system, Eq (3), to calculate the velocity.

Balancing tensor diffusivity (Gresho et al. [2]) was used as an additive correction to the diffusion matrix that balances the negative diffusion induced by the explicit Euler time integration. Also, hourglass correction was added to the diffusion matrix to damp any zero-energy modes that may be present because of the reduced integration scheme (Goudreau and Hallquist [3] and Gresho et al. [2]). To reduce the computational effort in the evaluation of the advection term, a "centroid advection velocity" simplification was used, as was done by Gresho et al. [2].

The temperature was solved independent of the flow equations. The thermal transport equation is

$$
\rho C_{v}\left(\frac{f T}{f t}+\vec{u} ? T\right)=? \kappa T+q
$$

where $T$ is the temperature, $t$ is time, $\kappa$ is the thermal conductivity, $\rho$ is the fluid density, $C_{v}$ is the heat capacity, and $q$ is the volumetric heat generation rate. This equation was also solved with the Galerkin FEM using tri-linear basis functions for the temperature and second-order Gaussian quadrature for the spatial integration. A fully implicit (backward-Euler) time-integration scheme was used. Although the code allows subcycling of the thermal diffusion equation, a single temperature solve was performed for 
each solution of the flow equations for all cases presented here. The temperature was fed back into the flow equations via a Boussinesq approximation for the buoyancy force,

$f_{\text {buoyanncy }}=\vec{g}\left(1-\beta\left(T-T_{0}\right)\right)$,

which was added to $f$ in $\mathrm{Eq}$ (2), where $g$ is the gravitational acceleration, $\beta$ is the coefficient of thermal expansion, and $T_{0}$ is a reference temperature.

The above matrices were assembled using the Finite Element Interface (FEI) [4] developed by Sandia National Laboratories in collaboration with Lawrence Livermore National Laboratory (LLNL). The system of equations was solved using the HYPRE [5] parallel solver package developed at LLNL's Center for Applied Scientific Computing (CASC). The above library packages allow the use of many advanced iterative linear solvers and preconditioners designed for efficient matrix solutions on massively parallel computer systems. The flow equations were solved using the conjugate gradient (CG) solver with parallel sparse approximate inverse (ParaSails) preconditioning. The thermal equations were solved using the generalized minimal residual (GMRES) solver with diagonal preconditioning. All of the computations used a convergence criteria of $1.0 \times 10^{-12}$ for the relative norm.

\section{Results}

An enclosed cavity with aspect ratio (height/width) of 8 was the focus of the current investigation. A temperature gradient was prescribed across the cavity, generating a buoyancy force, and driving the flow. A constant hot temperature, $T_{H}$, was imposed on the left $(x=0)$ vertical wall, and a cold temperature, $T_{C}$, was set on the right $(x=W)$ vertical wall. Insulated (i.e. no heat flux, $f T / f n=0$ ) boundary conditions were applied on 
the top and bottom horizontal walls of the cavity. No-slip $(u=v=0)$ boundary conditions were applied to the velocity on all walls. To solve this two-dimensional problem using our three-dimensional code, a single element was used in the out-of-plane (z) direction, and all z-direction velocities were set to zero. To remove the zero eigenvalues from the flow matrix, the pressure in two adjacent elements near the center of the cavity were pinned to zero. The flow was initially at rest, and the temperature was initialized to the average temperature of the hot and cold walls.

Although the code obtained solutions using dimensional units, all data presented here is in non-dimensional form to allow the values to be compared with the results of other investigators. The non-dimensional values use the characteristic length $W$ (cavity width), velocity $U=\sqrt{g \beta W\left(T_{H}-T_{C}\right)}$, time scale $\tau=W / U$, and pressure $\widetilde{P}=\rho U^{2}$. The non-dimensional temperature is defined as $\theta=\frac{T-1 / 2\left(T_{H}+T_{C}\right)}{T_{H}-T_{C}}$.

The temperatures and fluid properties were set such that the Rayleigh number (Ra) was $3.4 \times 10^{5}$ and the Prandtl number (Pr) was 0.71 .

The solution was calculated for two grids. A coarse grid with 30x100 elements and a fine grid of 60x200 elements were used. A third grid of 90x300 elements is currently being run, but there are no results at this time. The grid spacing was graded with a 3:1 ratio from the center of the cavity to the wall. The computations were performed in parallel on the IBM based ASCII Blue-Pacific computer at LLNL. The coarse grid was computed using 4 processors and required approximately 5 seconds of 
compute time (wall clock) per time step. The fine grid took approximately 10 seconds per time step using 12 processors.

\subsection{Point Data}

During the flow solution, time-history data at five points were reported at each time step. There was a point located near each of the four corners of the cavity and one was near the center of the hot wall. They are identified in non-dimensional coordinates as

\begin{tabular}{lll} 
point & $\mathrm{x} / \mathrm{W}$ & $\mathrm{y} / \mathrm{W}$ \\
\hline 1 & 0.1810 & 7.3700 \\
2 & 0.8190 & 0.6300 \\
3 & 0.1810 & 0.6300 \\
4 & 0.8190 & 7.3700 \\
5 & 0.1810 & 4.0000
\end{tabular}

Time-history plots of the non-dimensional temperature, $\theta$, are found in Figure 1, for the fine grid. After some initial transients in the solution, the flow reached a stationary state where the temperature exhibited periodic oscillations about a mean temperature value.

Table 1 provides a summary of the time-history data for the two grid resolutions. For each variable presented in the table, the time-averaged value is given along with the amplitude and period of the oscillations. The amplitude is defined as the peak-to-valley amplitude. The time average, of a generic variable $\phi$, was computed as $\bar{\phi}=\frac{1}{T}{ }_{0}^{T} \phi(t) d t$ where $T$ represents the total period of time for which the average was computed. For all data presented here, the average was integrated over 10 complete cycles near the end of the calculation. 
For each data point $i$, the table presents information for the velocity in the $x$ direction $\left(u_{i}\right)$, the velocity in the $y$ direction $\left(v_{i}\right)$, the temperature $\left(\theta_{i}\right)$, the vorticity $\left(\omega_{i}=\frac{f v}{f x}-\frac{f u}{f y}\right)$, and the pressure differences $\left(\Delta P_{i j}=P_{i}-P_{j}\right)$. It is observed that the two grids give similar answers for the average values, but very different results are obtained for the amplitude and period. The consistent values obtained for the period indicate a single dominant frequency is present within the flow field. The tabulated data reveal that the computations on both grids exhibit a very skew-symmetric flow field. As a measure of the skew-symmetry in the temperature field, the table presents the skewness $\left(\varepsilon_{i j}=\theta_{i}+\theta_{j}\right)$, which is found to be very small for both meshes.

\subsection{Global Data}

In addition to the point data presented above, global data is given in Table 2. Here the square root of the kinetic energy is used to provide a measure of the average velocity in the cavity. The velocity metric is

$\hat{u}=\sqrt{\frac{1}{2 A} \quad \vec{u} ? \vec{u} d A}$

where $A$ is the total area of the cavity. Little difference is found between grids for both the average value and the amplitude. The values for period reveal that the same dominant frequency that was seen in the point data is also present in the velocity metric.

\section{Conclusions}

Although it appears that the average value for the velocity and temperature data can be fairly accurately calculated using a coarse grid, the time-accurate statistics such as 
oscillation amplitude and frequency appear highly grid dependent. At this point, it is unknown how much grid refinement is required to accurately obtain these values. This is currently being investigated.

\section{Acknowledgements}

This work was performed under the auspices of the U.S. Department of Energy by Lawrence Livermore National Laboratory under contract no. W-7405-Eng-48.

\section{References}

[1] Christon MA, Gresho PM, Sutton SB, "Computational Predictability of Natural Convection Flows in Enclosures", Special Session, First M.I.T. Conference on Computational Fluid and Solid Mechanics, Massachusetts Institute of Technology, Cambridge, MA, June 12-14, 2001.

[2] Gresho PM, Chan ST, Lee RL, Upson CD, “A Modified Finite Element Method for solving the Time-Dependent, Incompressible Navier-Stokes Equations Part 1: Theory", International Journal for Numerical Methods in Fluids, 1984, Vol. 4, No. 6, pp. 557-598.

[3] Goudreau GL, Hallquist JO, "Recent Developments in Large-Scale Finite Element Lagrangian Hydrocode Technology", Computational Methods in Applied Mechanics and Engineering, 1982, Vol. 33, No. 1-3, pp. 725-757.

[4] Clay RL, Mish KD, Otero IJ, Taylor LM, Williams AB, “An Annotated Reference Guide to the Finite-Element Interface (FEI) Specification Version 1.0”, 
Technical Report SAND99-8229, Sandia National Laboratories, Livermore, CA, Jan. 1999.

[5] Chow E, Cleary AJ, Falgout RD, Lambert M, Tong C, Walker D, Yang UM, "hypre, high performance preconditioners: User’s Manual, v.1.2.0", Technical Report UCRL-MA-137155-DR, Lawrence Livermore National Laboratory, Livermore, CA, Aug. 2000. 
Figures and Tables

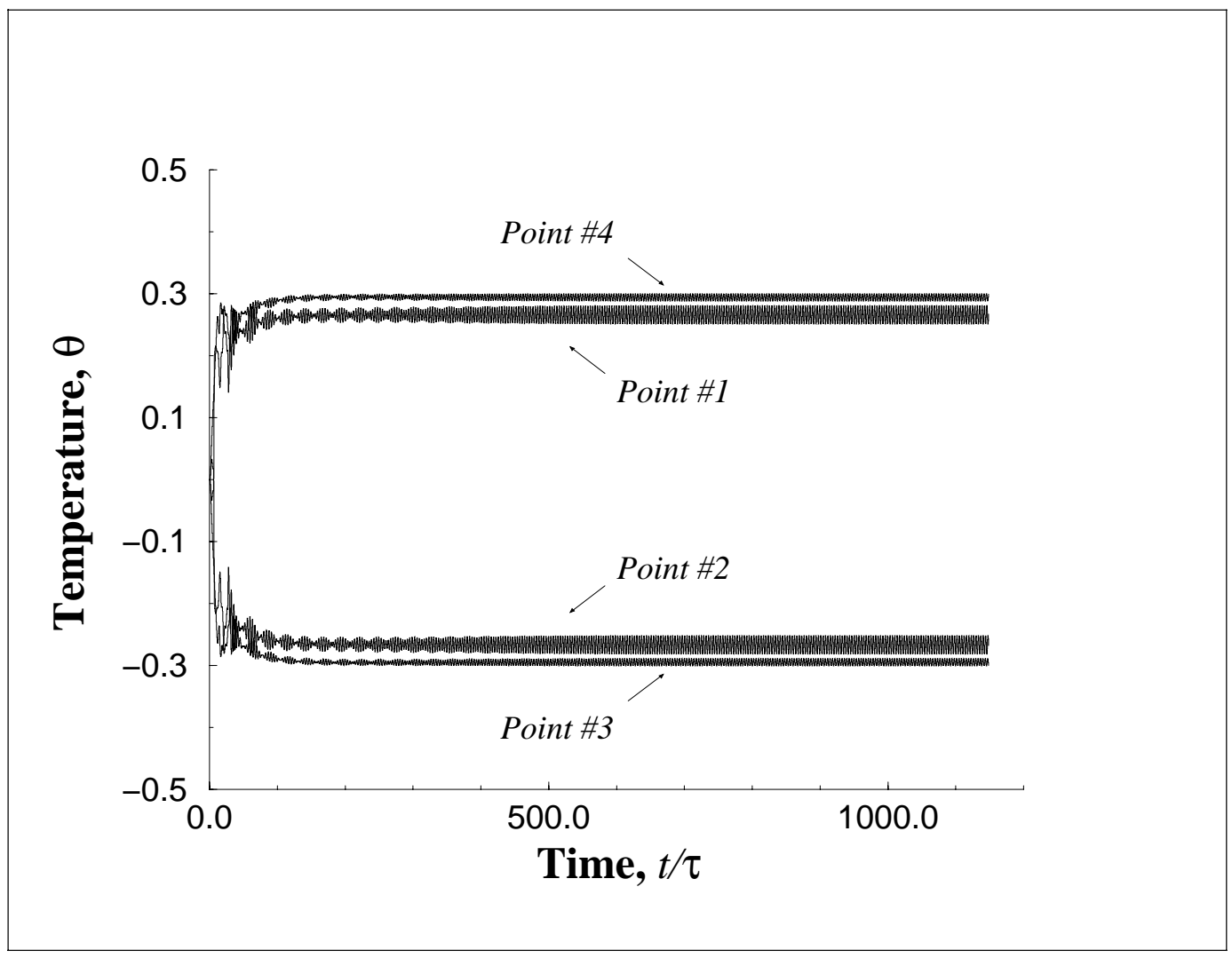

Figure 1: Time-history plot of temperature, calculated using a grid of 60x200 elements. 


\begin{tabular}{|c|c|c|c|c|c|c|}
\hline \multirow[b]{2}{*}{ Quantity } & \multicolumn{3}{|c|}{$\begin{array}{c}\text { Grid Resolution: } 30 \times 100 \\
\text { Time Duration: } 43.4 \\
\text { Steps per Period: } 75\end{array}$} & \multicolumn{3}{|c|}{$\begin{array}{c}\text { Grid Resolution: } 60 \times 200 \\
\text { Time Duration: } 35.2 \\
\text { Steps per Period: } 219\end{array}$} \\
\hline & Average & Amplitude & Period & Average & Amplitude & Period \\
\hline X-Velocity & & & & & & \\
\hline$u_{1}$ & $5.55 \mathrm{e}-2$ & $9.07 e-3$ & 4.34 & $5.53 e-2$ & $3.94 \mathrm{e}-2$ & 3.52 \\
\hline$u_{2}$ & $-5.55 e-2$ & $9.07 e-3$ & 4.34 & $-5.53 e-2$ & $3.94 \mathrm{e}-2$ & 3.52 \\
\hline$u_{3}$ & $-3.19 e-2$ & $3.10 \mathrm{e}-3$ & 4.34 & $-3.25 e-2$ & $1.077 \mathrm{e}-2$ & 3.52 \\
\hline$u_{4}$ & $3.19 \mathrm{e}-2$ & $3.10 \mathrm{e}-3$ & 4.34 & $3.25 \mathrm{e}-2$ & $1.077 \mathrm{e}-2$ & 3.52 \\
\hline$u_{5}$ & $2.65 \mathrm{e}-4$ & $3.68 \mathrm{e}-4$ & 4.34 & $2.59 \mathrm{e}-4$ & $2.56 \mathrm{e}-3$ & 3.52 \\
\hline Y-Velocity & & & & & & \\
\hline$v_{1}$ & 0.459 & $1.556 \mathrm{e}-2$ & 4.34 & 0.462 & $5.79 e-2$ & 3.52 \\
\hline$v_{2}$ & -0.459 & $1.556 \mathrm{e}-2$ & 4.34 & -0.462 & $5.79 \mathrm{e}-2$ & 3.52 \\
\hline$v_{3}$ & 0.367 & $2.18 \mathrm{e}-3$ & 4.34 & 0.382 & $9.27 \mathrm{e}-3$ & 3.52 \\
\hline$v_{4}$ & -0.367 & $2.18 \mathrm{e}-3$ & 4.34 & -0.382 & $9.27 \mathrm{e}-3$ & 3.52 \\
\hline$v_{5}$ & 0.421 & $1.469 \mathrm{e}-3$ & 4.34 & 0.416 & $7.34 \mathrm{e}-3$ & 3.52 \\
\hline Temperature & & & & & & \\
\hline$\theta_{1}$ & 0.267 & $6.86 \mathrm{e}-3$ & 4.34 & 0.266 & $3.02 \mathrm{e}-2$ & 3.52 \\
\hline$\theta_{2}$ & -0.267 & $6.86 \mathrm{e}-3$ & 4.34 & -0.266 & $3.02 \mathrm{e}-2$ & 3.52 \\
\hline$\theta_{3}$ & -0.297 & $2.70 \mathrm{e}-3$ & 4.34 & -0.294 & $1.198 \mathrm{e}-2$ & 3.52 \\
\hline$\theta_{4}$ & 0.297 & $2.70 \mathrm{e}-3$ & 4.34 & 0.294 & $1.198 \mathrm{e}-2$ & 3.52 \\
\hline$\theta_{5}$ & $1.034 \mathrm{e}-3$ & $5.22 \mathrm{e}-4$ & 4.34 & $-5.90 e-4$ & $3.19 \mathrm{e}-3$ & 3.52 \\
\hline Skewness & & & & & & \\
\hline$\varepsilon_{12}$ & $2.12 \mathrm{e}-7$ & $3.13 \mathrm{e}-6$ & 4.34 & $2.31 \mathrm{e}-6$ & $1.048 \mathrm{e}-5$ & 3.52 \\
\hline Vorticity & & & & & & \\
\hline$\omega_{1}$ & -2.42 & 0.1971 & 4.34 & -2.59 & 0.641 & 3.52 \\
\hline$\omega_{2}$ & -2.42 & 0.1971 & 4.34 & -2.59 & 0.641 & 3.52 \\
\hline$\omega_{3}$ & -1.075 & $3.38 \mathrm{e}-2$ & 4.34 & -1.266 & 0.1586 & 3.52 \\
\hline$\omega_{4}$ & -1.075 & $3.38 \mathrm{e}-2$ & 4.34 & -1.266 & 0.1586 & 3.52 \\
\hline$\omega_{5}$ & -4.67 & $8.16 \mathrm{e}-3$ & 4.34 & -4.51 & $6.78 \mathrm{e}-2$ & 3.52 \\
\hline$\underline{\text { Pressure }}$ & & & & & & \\
\hline$\Delta P_{14}$ & $-2.68 e-3$ & $3.73 e-3$ & 4.34 & $-2.30 \mathrm{e}-3$ & $1.529 \mathrm{e}-2$ & 3.52 \\
\hline$\Delta P_{35}$ & -1.180 & $5.00 \mathrm{e}-4$ & 4.34 & -1.163 & $7.62 \mathrm{e}-3$ & 3.52 \\
\hline$\Delta P_{51}$ & -2.19 & $3.34 \mathrm{e}-3$ & 4.34 & -2.19 & $1.775 \mathrm{e}-2$ & 3.52 \\
\hline
\end{tabular}

Table 1: Point data for two grid resolutions was tabulated from the time-history plots at five points within the cavity. 


\begin{tabular}{|c|ccc|ccc|}
\hline & \multicolumn{3}{|c|}{ Grid Resolution: 30x100 } & \multicolumn{3}{c|}{ Grid Resolution: 60x200 } \\
& \multicolumn{2}{|c|}{ Time Duration: 43.4} & \multicolumn{3}{c|}{ Time Duration: 35.2} \\
Steps per Period: 75 & \multicolumn{2}{c|}{ Steps per Period: 219} \\
Quantity & Average & Amplitude & Period & Average & Amplitude & Period \\
\hline$\hat{u}$ & 0.239 & $3.68 \mathrm{e}-5$ & 4.34 & 0.239 & $3.84 \mathrm{e}-5$ & 3.52 \\
\hline
\end{tabular}

Table 2: The square root of the kinetic energy provides a global measure of the average velocity within the cavity. 